García-Martínez, B., Lozano-Martínez, J. \& Cerezo-Maiquez, M.C. (2021). Educación intercultural-inclusiva desde el patrimonio cultural: la voz del alumnado. Revista Electrónica Interuniversitaria de Formación del Profesorado, 24(3), 185-201.

\title{
Educación intercultural-inclusiva desde el patrimonio cultural: la voz del alumnado
}

Bienvenida García Martínez, Josefina Lozano Martínez, Mari Carmen Cerezo Maiquez.

Universidad de Murcia

\section{Resumen}

El presente estudio se centra, principalmente, en investigar la percepción que el alumnado de Educación Primaria e Infantil, de una localidad de la Región de Murcia, tiene de la enseñanza del patrimonio cultural desde actividades complementarias al currículo inscritas dentro de la educación no formal. El programa de intervención se desarrolla en el Museo Etnológico de la Huerta de Murcia, en base a talleres y estrategias que favorecen la adquisición de competencias curriculares señaladas en la normativa educativa vigente y fomentan la educación intercultural e inclusiva. Como instrumento de recogida de información se ha utilizado un cuestionario. También se ha realizado un análisis de contenido desde lo recogido en la observación participante y el diario de campo, dado el carácter etnográfico del estudio. Los resultados indican que las actividades formativas en torno al Patrimonio Cultural desde la enseñanza no formal, son un complemento, de cara al alumnado, muy eficaz para la adquisición de conocimientos dentro de una educación intercultural e inclusiva y que favorece la construcción de sociedades más democráticas.

\section{Palabras clave}

Educación patrimonial; diversidad; educación intercultural e inclusiva; enseñanza no formal.

\section{Contacto:}

Bienvenida García Martínez, bienvenida.garcia@um.es. Facultad de Educación, Campus de Espinardo, 30100, Murcia. Este artículo forma parte de un proyecto doctoral de investigación. 


\section{Intercultural - inclusive education from the cultural heritage: the voice of the students}

\section{Abstract}

The present study focuses, mainly, on investigating the perception that primary and infant education students from a town in the Murcia region, have of the teaching of cultural heritage from activities that complement the curriculum, registered within non-formal education. The intervention program takes place in the Ethnological Museum of La Huerta from Murcia, based on workshops and strategies that favor the acquisition of curricular competences indicated in the current educational regulations and that benefit intercultural and inclusive education. As an instrument for collecting information, a questionnaire has been used. A content analysis has also been carried out from what was collected in the participant observation and the field diary, given the ethnographic nature of the study. The results indicate that the training activities around Cultural Heritage from non-formal education are a complement, for student, very effective for the acquisition of knowledge within an intercultural and inclusive education and favor the construction of more democratic societies.

\section{Key words}

Heritage education, variety, intercultural and inclusive education, non-formal education.

\section{Introducción}

La educación es la base sobre la que se construyen las sociedades, que capacita al individuo para vivir en ellas. Por ello, se deberían adoptar medidas innovadoras y efectivas y aunar los esfuerzos en ese enfoque (Leiva y Borrero, 2011; Lozano y Cerezo, 2020). La situación social y cultural actual requiere desarrollar metodologías que fomenten la convivencia, poniendo en valor la existencia de culturas concretas con una diversidad de costumbres, con sus aciertos y sus errores, que deben aprender a convivir dialogando (Lozano, Ballesta, Cerezo, Luque, Castillo, 2017). Debemos apostar por una educación capaz de transformar la diversidad en algo positivo, que lleve al entendimiento mutuo y a la inclusión social (Pérez y Serrate, 2013) enseñando el valor de los que son diferentes, fomentando la diversidad y garantizando el éxito escolar (Etxeberria, Karrera y Murua, 2010). De este modo, la educación intercultural e inclusiva aparece como el enfoque más idóneo, el más ético para lograr dichos objetivos (Arnaiz, 2012; Casanova, 2011; Escudero y Martínez, 2011). Por consiguiente, siguiendo a Cerezo (2015), nos planteamos si los centros educativos aprovechan la realidad multicultural actual para favorecer dicha educación intercultural, o si sería importante la cohesión de procesos de aprendizaje formal y no formal (Ainscow, Dyson, Goldrick y West, 2012). 
Entendemos por cultura como una construcción del ser humano vinculado con su entorno natural y la historia de un lugar, que constituye su memoria colectiva. Pero las culturas no son estancas, sino que evolucionan y se desarrollan influidas por otras a lo largo de la historia (Osorio Franco, 2015). Así, observamos la importancia de crear vínculos entre presente y pasado y otorgar un lugar preeminente a la memoria y a la historia, elementos constitutivos de nuestro patrimonio cultural. Desde esta perspectiva, consideramos que la educación debe favorecer el estudio del patrimonio cultural, acercando a niños y jóvenes al conocimiento de diferentes manifestaciones artísticas y culturales de su entorno que puede fomentar el aprecio y valoración hacia estas y contribuir a la educación intercultural e inclusiva. Estos aprendizajes deberían comenzarse a edades más tempranas y desde otras perspectivas metodológicas más sencillas y eficaces (Llonch, 2011).

En España, a partir de 2003, surge una corriente de investigación que toma como referentes la didáctica del patrimonio y los estudios de museos (Fontal-Merillas e IbáñezEtxeberria, 2017). La educación patrimonial puede lograr que sus propietarios simbólicos los ciudadanos- quieran hacerse cargo y transmitirlo, haciéndolo de modo respetuoso con los bienes patrimoniales y con las sociedades que los han conservado y transmitido a lo largo de la historia (Fontal Merillas e Ibáñez Etxeberria, 2015). En este sentido, pese a que la escuela es el principal vehículo para difundir la cultura, la educación no formal, como la que se está desarrollando en los museos, puede desempeñar un papel clave para fomentar los principios de interculturalidad e inclusión, factores necesarios para gestionar la diversidad.

La generación de redes de apoyo y colaboración se presentan como importantes estrategias con las que dar una respuesta inclusiva a la diversidad (Echeíta, Simón, Sandoval y Monarca, 2013). No obstante, tal como señala Ainscow (2015), pocas escuelas las ponen en marcha, dada su complejidad. En línea de lo aportado por autores como Lozano, Ballesta, Castillo y Cerezo, (2018), consideramos que la vinculación de la escuela con su entorno y la colaboración entre esta y otros agentes socioeducativos del territorio es fundamental $\mathrm{y}$, desde el municipio, se puede contribuir a mejorar los procesos de enseñanza-aprendizaje de los alumnos y al desarrollo socioeducativo de los mismos. Se deben concentrar los esfuerzos para favorecer la cultura colaborativa (Sales, Moliner, Amiama y Lozano, 2018): Interconectar al mayor número de agentes, establecer redes sociales de apoyo (Escudero y Martínez, 2011), fomentar la participación de las instituciones sociales del entorno de los centros escolares, hacerlos más democráticos e inclusivos (Arnaiz, 2012; Arnaiz, De Haro y Azorín, 2018; Brik, Sebring, Allensworth, Luppescu y Easton, 2010). Las instituciones escolares deberían garantizar el aprendizaje crítico, así como, las relaciones horizontales y transformadoras, un liderazgo compartido entre escuela y otros agentes del territorio, con un mismo propósito (Anderson y Minke, 2007; Traver, Sales, Moliner, Sanahuja y Gil, 2018), dado que dicha colaboración facilita el aprendizaje del alumnado y beneficia a la escuela (Mager y Nowak, 2012). Esto implica poner en marcha metodologías didácticas y actividades que cohesionen los procesos de aprendizajes formales y no formales (Ainscow, Dyson, Goldrick y West, 2012; Cabalé-Miranda y Rodríguez-Pérez de Agreda, 2017).

La presente investigación forma parte de otra más extensa, pero en este caso recogemos una parte de la misma donde nos planteamos como objetivos averiguar cómo 
valoran los alumnos este tipo de enseñanza no formal desde la implementación de un programa de intervención, a fin de:

- Constatar si las actividades educativas no formales desarrolladas en ámbitos museísticos, para el estudio del patrimonio cultural, complementan a la educación reglada y favorecen la adquisición de conocimientos.

- Averiguar si las actividades del programa de intervención contribuyen a fomentar prácticas inclusivas entre el alumnado.

- Corroborar si la enseñanza-aprendizaje del patrimonio cultural más próximo, favorece el aprendizaje crítico y significativo para la construcción de una ciudadanía más democrática.

\section{Metodología}

\section{Población y participantes}

La investigación se ha realizado en el municipio de Alcantarilla perteneciente a la Comunidad Autónoma de la Región de Murcia, de un nivel económico y cultural que oscila entre medio y medio-bajo, que cuenta con 42048 habitantes, siendo la población de origen extranjero un total de 3893 personas, lo que supone el $9.28 \%$ del total. En la actualidad hay una población escolar de 4576 alumnos de Educación Infantil, Primaria y Educación Especial, y cuenta con once centros educativos donde se estudian estos ciclos, dos de ellos concertados. El tipo de muestreo fue no probabilístico casual, ya que participaron los alumnos que tomaron parte en las actividades propuestas en el Museo Etnológico de la Huerta, en el momento de efectuar la recogida de información, tras solicitar el permiso correspondiente al profesorado.

El número de participantes ha sido de 1165 alumnos/as y 69 docentes (en este caso nos centramos solo en el alumnado), de Educación Infantil y Primaria de 7 centros de Alcantarilla, a lo largo del curso 2019/2020, fechas en las que ha tenido lugar el trabajo de campo de la investigación. El nivel de confianza obtenido, teniendo en cuenta el número de participantes ( 1165 alumnos/as), de un total de 4576, matriculados en el municipio de Alcantarilla en el curso 2019/2020, resulta del $95 \%$ con un error de \pm 3 , lo que nos proporciona un tamaño muestral adecuado, para realizar un estudio generalizado en dicha población. De estos participantes un $25.39 \%$ son de origen extranjero, como podemos comprobar en la Figura 1. 
Figura 1

Gráfico de la variable nacionalidad del alumnado

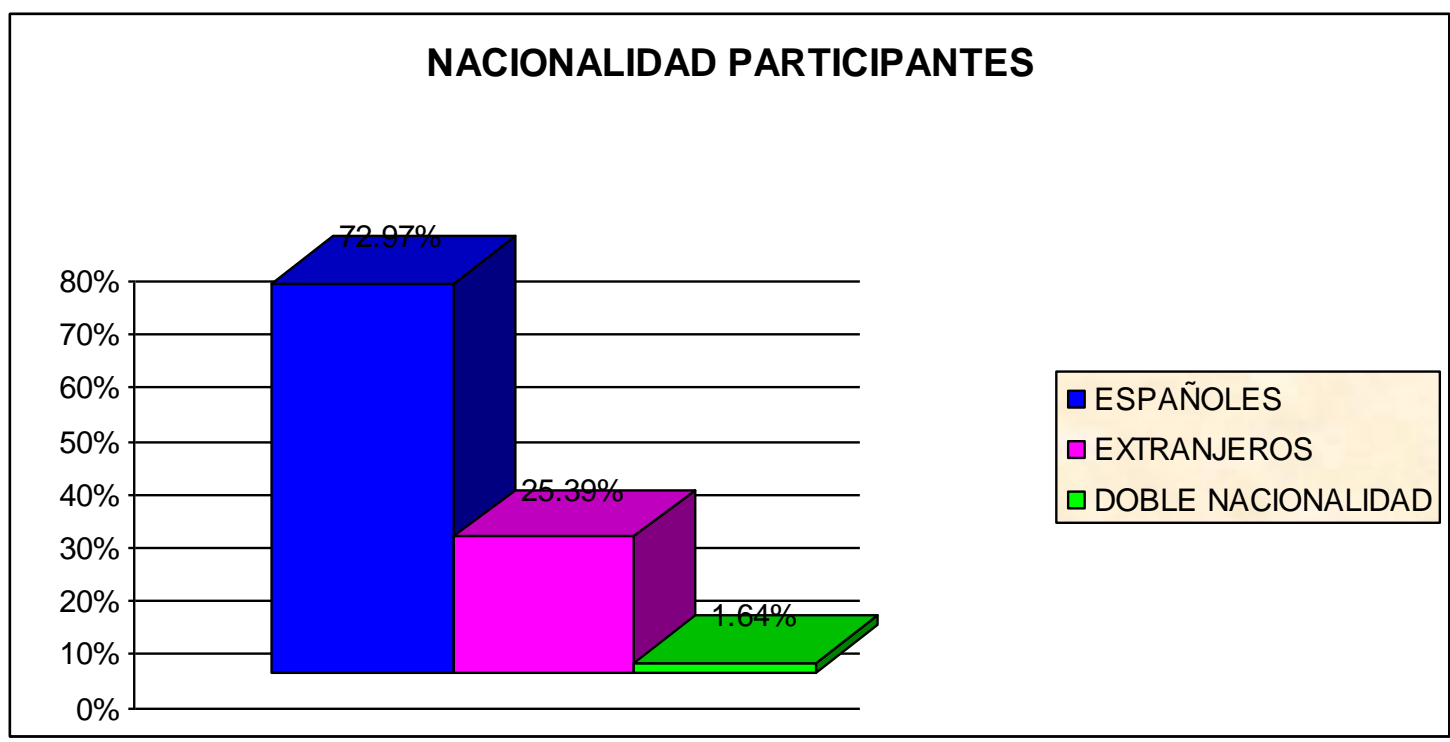

Además, en las actividades que nos sirvieron como vía para implementar esta investigación, han participado como monitores, seis chicos y chicas que, en la actualidad están cursando ciclo formativo de jardinería en el Colegio de Educación Especial Eusebio Martínez de esta localidad, así como dos de sus profesores. También ha colaborado una graduada de Educación Primaria en la especialidad de Educación Física, un profesor de Música que, a su vez, es licenciado en Psicología, una técnico en gestión cultural y una licenciada en Historia del Arte y máster en Antropología Social.

\section{Instrumentos de recogida y análisis de la información}

En esta investigación se combina la metodología cuantitativa -cuestionario- y cualitativa -observación participante-. Por tanto, está enfocada desde un diseño mixto de investigación. Los instrumentos que consideramos más útiles han sido el cuestionario y el Diario de Campo (DC).

Hemos utilizado la técnica de la Observación Participante (OP), para recoger la información de lo observado y los comentarios realizados por los alumnos (A), de los diferentes Centros (C) que han participado. Estas codificaciones adjuntas entre paréntesis se utilizarán en los resultados; apoyándonos, además, en archivos visuales (imágenes y vídeos), instrumentos muy utilizados en investigaciones etnográficas (García-Pérez, 2018), que pueden combinarse y servir de complemento a otras técnicas de recogida de datos tanto cualitativas como cuantitativas (Hammersle y Atkinson, 2007; Creswell, 2005).

El cuestionario nos ha permitido conocer por escrito las opiniones y pensamientos de los encuestados, tal como señalan Colas, Buendía y Hernández (2009), nos ha facilitado la recogida y análisis de una muestra amplia con un carácter ambivalente, cuantitativo y cualitativo (Cerezo, 2015). 
Los alumnos cumplimentaron un cuestionario (Ver Anexo I), tras las actividades realizadas en el Museo de la Huerta, compuesto por 12 ítems, si bien, los dos últimos, van dirigidos, específicamente, al alumnado extranjero, siendo la última una pregunta abierta. Los ítems, de tipo Likert están distribuidos en tres dimensiones, que actúan como variables criterio. Los ítems cerrados se miden con una escala numérica de cuatro grados (1. Malo/Nunca/Muy poco; 2. Regular/Casi Nunca/Poco; 3. Bueno/A veces/Regular; 4. Muy buena/Mucho/Siempre). Fue validado por varios expertos: la directora de Museo Etnológico de la Huerta de Alcantarilla, una profesional de la gestión cultural, un maestro, una psicólogo-terapeuta especialista en niños con necesidades especiales, dos profesoras de universidad especialistas en Didáctica y Organización Escolar y Métodos de Investigación. Atendiendo a la fiabilidad del cuestionario (Coeficiente Omega) es de 0.82. Hemos utilizado este coeficiente dado el número reducido de ítems que compone el instrumento y por el por el número de ítems de las cuestiones ni por el número de respuestas dadas. Un nivel aceptable de este coeficiente se encuentra entre .70 y .90 (Campo Arias \& Oviedo, 2008).

Para el análisis de los datos se ha utilizado el programa Statistical Package for the Social Sciences(SPSS.24), el cual permite la realización de técnicas descriptivas e inferenciales. Para el análisis de datos cualitativos se han utilizado todas aquellas frases, párrafos y fragmentos recogidos de la observación participante anotados en el diario de campo, con todo ello se ha hecho un análisis de contenido en función del objeto de estudio del cuestionario.

Este cuestionario también fue adaptado a través de pictogramas para que pudiera ser respondido por los niños/as de los cursos de Educación Infantil y primeros niveles de Educación Primaria (Ver Anexo II).

\section{Programa de Intervención y procedimiento}

Se elabora un programa de intervención, desarrollado en el Museo Etnológico de la Huerta de Murcia, que sirve de canal para implementar la investigación, así como, para la elección del método y técnicas de investigación. Actividades desde la enseñanza no formal, utilizadas como estrategias para adquirir estándares de aprendizaje que favorezcan la adquisición de competencias contempladas en el currículo escolar, de una manera transversal. En especial, se busca fortalecer aquellas competencias del patrimonio cultural que instan a los alumnos a formarse en una educación intercultural e inclusiva y de respeto a la diversidad. Dichas actividades han sido adaptadas a cada tramo educativo, utilizando recursos como un cuento, para los más pequeños; y un teatrillo que los alumnos mayores escenifican. Los centros eligen entre diferentes talleres que los alumnos realizan en equipos:

- Laboratorio de Cocina.

- Taller de Arqueología.

- Taller de Danzas y Músicas del mundo, incluida la jota murciana.

- Taller Aula de la Naturaleza. 


\section{Resultados}

Para interpretar los resultados, a partir los datos aportados por los alumnos, hemos realizado el estudio de cada una de las preguntas correspondientes a cada una de las Dimensiones I, II y III del cuestionario, relacionadas con los objetivos descritos. Además del análisis descriptivo, hemos realizado un análisis inferencial que nos permite comprobar si existe algún tipo de relación entre los distintos ítems del cuestionario con los datos del perfil de la muestra. Recogemos algunos valores de las correlaciones y se señalan las diferencias significativas $(p<.05)$; es decir, aquellas que parece influir las variables estudiadas entre ellas. Para facilitar la lectura, solo vamos a describir las que indican una diferencia significativa entre las variables: titularidad del centro, grupo-ciclo formativo, género, nacionalidad, grupo de país con algunas de las preguntas del cuestionario incluidas en cada una de las dimensiones. Debemos anotar que, aunque las variables titularidad del centro y grupo-ciclo formativo no se recogen en el cuestionario, se ha hecho acopio de estos datos en el diario de campo. Detectamos mayor número de alumnos inmigrantes en colegios de titularidad pública, $28.39 \%$, frente a un $17.95 \%$ en los concertados.

En la DIMENSIÓN I, las preguntas 1 y 2 del cuestionario están relacionadas con el primer objetivo: Constatar si las actividades educativas no formales desarrolladas en ámbitos museísticos, para el estudio del patrimonio cultural, complementan a la educación reglada y favorecen la adquisición de conocimientos. Respecto de las cuestiones contempladas observamos que en la primera pregunta (P.1), ¿Qué te ha parecido esta actividad fuera del colegio realizada en el museo?, el $95.62 \%$ del alumnado la valoran como buena o muy buena. Detectamos diferencias significativas entre las contestaciones dadas según los diferentes niveles o ciclos formativos; así contestan muy buena/buena (p.00o), el 6.9\% del alumnado de los cursos de Infantil, el $35.5 \%$ del primer tramo de Primaria y el $53.5 \%$ de los de segundo tramo. Ateniéndonos a la variable nacionalidad (p.001), el 69.5\% de los alumnos españoles responden muy buena/buena a esta pregunta y el $25.7 \%$ de los de origen extranjero. Si bien, debemos señalar que, el número de alumnos españoles participantes ha sido de 870 frente a 295 extranjeros.

Sobre la segunda cuestión (P.2), ¿Piensas que con esta actividad se puede aprender más fácilmente que con los libros y demás materiales que se utilizan en las aulas?, más del $93.65 \%$ del alumnado ha contestado que siempre o a veces. Encontramos algunas diferencias significativas entre las opiniones dadas por los alumnos a esta cuestión según la titularidad de los centros. Los de los públicos contestan siempre/a veces (p.009) en un $78.9 \%$ y el $24.8 \%$ de los concertados. Según el nivel formativo contestan siempre/a veces (p.000), el 7\% de los niños y niñas de Infantil, el $33.5 \%$ del primer tramo y a un $53.1 \%$ del segundo.

Algunos comentarios hechos por los alumnos relacionados con este objetivo, recogidos en el diario de campo son:

- "Yo pensaba que iba a ser lo mismo que la última vez que vine a este museo" (DC, (1, A5).

- "Ahora me ha gustado mucho más que la última vez que vine” (DC. C2. A9). 
- “Cuando empezó la visita no entendía bien lo que nos estaban explicando, después, cuando fuimos haciendo las actividades, lo entendí todo" (DC. C1. A20).

En lo referente al segundo objetivo: Averiguar si las actividades llevadas a cabo desde la enseñanza no formal, como complemento de la educación reglada, contribuyen a fomentar prácticas inclusivas entre el alumnado, está relacionado con la DIMENSIÓN II del cuestionario (preguntas $\mathrm{P}_{3}, \mathrm{P}_{4}, \mathrm{P}_{5}, \mathrm{P} 6$ ). Así, a la tercera cuestión, ¿te gusta trabajar en equipo?, casi tres cuartas partes del alumnado, 73.99\%, respondió que siempre y un tercio dice a veces. Aquí observamos diferencias significativas respecto a la titularidad dado que el $81 \%$ del alumnado de centros públicos contesta afirmativamente (p.000) y solo el $15.7 \%$ de los concertados. También en cuanto al ciclo: contestan siempre/a veces (p.000) el $6.8 \%$ de Infantil, el $35.7 \%$ del primer tramo y un $54.4 \%$ del segundo.

Cuando se les pregunta: ¿crees que trabajar en equipo ayuda a aprender mientras nos divertimos? (P.4), tres cuartas partes, $74.25 \%$, opina que siempre y casi la tercera parte afirma que $a$ veces. Comprobamos diferencias significativas ya que el $80.1 \%$ de los alumnos de centros públicos contestan siempre/a veces (p.003), y el $15.3 \%$ de los de concertados. Según el ciclo formativo (p.000), el porcentaje de respuesta es del $6.9 \%$ en Infantil, el $35.3 \%$ del primer tramo y $53.2 \%$ del segundo.

Debemos señalar que, generalmente, los equipos se han formado siguiendo el orden de lista de clase. Las respuestas a la cuestión (P.5): si te toca ir en un equipo con compañeros que no son tus mejores amigos o amigas, ¿te importa?, los resultados nos indican que algo más de la mitad, 59.4\%, contesta que muy poco o poco, mientras que un $39.32 \%$ prefiere formar parte de equipos en los que estén sus amigos. En esta respuesta también se observan diferencias según del nivel de los alumnos, así, contestan mucho/regular (p.000) el 1.8\% los de Infantil, el 13.9\% del primer tramo de Primaria y el $23.6 \%$ del segundo.

En la siguiente cuestión (P.6) que les planteamos: ¿crees que las actividades y salidas que se realizan fuera del centro escolar favorece conocer mejor a los compañeros?, más de la mitad, $57.68 \%$, opina que siempre y un tercio, $30.82 \%$, contesta que a veces. Aquí comprobamos diferencias significativas dependiendo de la titularidad el $76 \%$ de los alumnos de centros públicos opinan que siempre/a veces (p.000), y el 12.6\% de los de los concertados. También respecto al nivel (p.000) del alumnado: el 6.8\% de los niños y niñas de Infantil contestan afirmativamente, también el $31.7 \%$ del primer tramo y el $50.1 \%$ del segundo.

A continuación se explicitan algunas observaciones y comentarios de los niños y niñas que hacen referencia a este objetivo, recogidos en el Diario de Campo (DC).

- “Trabajar en equipo me gusta porque así ayudamos a los demás “(DC. C3. A12).

- " "Es más divertido cuando hacemos cosas juntos" (DC. C7. A15).

- " "Si puedo elegir, prefiero ir con mis amigos" (DC. C5. A12).

- Un niño africano, apenas se separó de una niña que andaba en silla de ruedas. Dada su complexión, se pasó casi toda la excursión empujándola (DC. C1. A18). 
- Otra niña que no se separaba de un niño con Síndrome de Down, estuvo ayudándole a excavar durante el taller de arqueología (DC. C7. A3).

- "Hacemos cosas con compañeros con los que no solemos relacionarnos habitualmente" (DC. C6. A5).

El último objetivo: Corroborar si la enseñanza-aprendizaje del patrimonio cultural más próximo, favorece el aprendizaje crítico y significativo para la construcción de una ciudadanía más democrática, se relaciona con las preguntas (P7, P8, P9 y P10) de la DIMENSIÓN III del cuestionario.

Los resultados plantean que más de la mitad, $53.22 \%$, del alumnado dice saber poco o muy poco sobre el patrimonio cultural (P.7) y el $25.58 \%$ afirma que regular. Se evidencias diferencias en cuanto a que el $37.1 \%$ de los alumnos de los centros públicos contesta mucho/regular (p.002), y el $7.6 \%$ de los concertados. En lo referente al nivel formativo, contestan afirmativamente (p.000) el 1.8\% de Infantil, un $17 \%$ de alumnos del primer tramo y el $25.9 \%$ de los del segundo.

Cerca del $80 \%$ opina gustarle mucho saber acerca de las tradiciones y costumbres de sus antepasados (P.8). Existen diferencias significativas respecto a esta pregunta dependiendo del nivel o ciclo (p.000), opina así el 6.1\% de Infantil, el 33.6\% del primer tramo y un 50.4\% del segundo. También según el género (p.002) y, aunque el número de niños y niñas participantes es bastante paritario, 569 niños frente a 592 niñas, comprobamos que responden afirmativamente un $47.4 \%$ de las niñas y un $42.4 \%$ de niños.

Sólo la tercera parte (37.08\%) reconoce haber oído hablar mucho o regular de otras culturas que habitaron en Murcia (P.9). Detectamos diferencias de respuesta según la titularidad (p.000): $57.7 \%$ de los públicos y el $8.2 \%$ de los alumnos de concertados. Igual ocurre con la variable nivel (p.000): $1.3 \%$ de Infantil contesta afirmativamente así como el $20.4 \%$ del alumnado del primer tramo y el $44.2 \%$ del segundo

Sin embargo, demuestran interés por el tema dado que casi a un $70 \%$ dice gustarle mucho adquirir conocimientos sobre otras culturas diferentes a la suya (P.10). Pese a que existen diferencias significativas en las contestaciones dependiendo de la titularidad (p.000): $65.4 \%$ de públicos y 14.7\% de los concertados. Diferencias también según nivel o ciclo (p.000): $6.51 \%$ de Infantil, un 33\% del primer tramo y el $41.4 \%$ del segundo.

Como hemos señalado, la última pregunta (P.11): ¿Has encontrado algún parecido entre estas costumbres y las de tu país de origen?, está dirigida sólo al alumnado extranjero.

De los 295 alumnos extranjeros participantes el 17.29\% afirma haber encontrado muchos parecidos, el $19.32 \%$ algunos parecidos, mientras el $11.86 \%$ dice encontrar pocos y el $17.63 \%$ ningún parecido. Los alumnos con doble procedencia dicen encontrar muchos parecidos el $10.53 \%$ y algunos el $36.84 \%$. Mientras que el resto dice haber encontrado pocos (10.53\%) o ningún parecido (15.79\%). En esta también se detecta diferencia significativa en las respuestas dadas por los alumnos según el grupo de país (p.000). Los alumnos que más parecidos han encontrado entre nuestras costumbres y las de sus países de origen han sido los procedentes de países africanos, $0.13 \%$, seguido de los latinoamericanos, $0.8 \%$ y los europeos, $0.5 \%$. 
Estos datos se constatan en algunos comentarios que han hecho los alumnos, recogido en el Diario de Campo (DC):

- "Mis abuelos me cuentan cosas de cómo vivían cuando eran niños” (DC. C3. A3).

- Como anécdotas: "un niño árabe y un hispanoamericano, hablaban de cómo los primeros dicen ¡Alaaa! cuando se sorprenden de algo y los otros dicen ¡Dios míooo!, refiriéndose a que era lo mismo (DC. C3. A7-A18).

Detectamos que son los alumnos procedentes de países árabes y latinoamericanos, los que más parecidos han encontrado con nuestras costumbres. Algunos alumnos marroquíes han señalado ciertos parecidos:

- "En mi país también se comen los caracoles" (DC. C1. A18).

“En mi país también se riegan los huertos-campos, de esta manera" (DC. C6. A12), refiriéndose al tradicional sistema de regadío de la huerta murciana.

\section{Discusión y conclusiones}

A continuación, desde la discusión de los resultados, iremos concluyendo y dando respuesta a los distintos objetivos planteados en la presente investigación. En primer lugar, se ha podido constatar que las actividades educativas no formales para el estudio del patrimonio cultural, desarrolladas en ámbitos museísticos, complementan a la educación reglada y favorecen la adquisición de conocimientos. Los resultados obtenidos nos hacen prever que, dada la motivación del alumnado desarrollado en la ejecución de los distintos talleres, se ha fomentado el conocimiento de este patrimonio cultural, que puede convertirse en un motor de arranque para, a partir de estos, impulsarse medidas educativas que favorezcan la adquisición de nuevos conocimientos y la cercanía a las costumbres propias del entorno (Escudero y Martínez, 2011; Sales, Moliner, Amiama y Lozano, 2018).

Comprobamos también, que las actividades llevadas a cabo desde la enseñanza no formal, como complemento de la educación reglada, contribuyen a fomentar prácticas inclusivas entre el alumnado. De acuerdo con Fontal- Merillas e Ibáñez-Etxeberria (2015) y Lucas-Palacios (2014), estimamos que la educación patrimonial, desde la enseñanza no formal, es un instrumento eficaz, no sólo para que los alumnos adquieran competencias y aprendan a respetarlo y conservarlo, sino porque se presenta como un instrumento idóneo para favorecer la educación intercultural e inclusiva y el respeto a la diversidad, dado que sirve tanto para favorecer las relaciones interpersonales como los aprendizajes (CarriónGútiez, 2015). Al igual que Alves y Pinto (2019); Fontal- Merillas e Ibáñez-Etxeberría (2015); entendemos que la enseñanza-aprendizaje del patrimonio no debe limitarse a los contenidos del currículo, al ámbito de las ciencias sociales exclusivamente, ni a aspectos conservacionistas, sino que deben aplicarse otros puntos de vista e interpretaciones, teniendo en cuenta la transversalidad de materias y contenidos, en los cuales el modelo educativo del patrimonio se centra en los sujetos, favoreciendo su reflexión y crecimiento personal, interconectándolos con su entorno más próximo. Lo más significativo de esta investigación es que en un taller, concretamente, en el de Aula de la Naturaleza, han colaborado como monitores, alumnos del Centro de Educación Especial Eusebio Martínez, de Alcantarilla, que en estos momentos están cursando ciclos formativos de jardinería. Este 
taller, que ha sido desarrollado por alumnos mayores con discapacidad intelectual, les ha permitido empoderarse, al tiempo que ha demostrado a los alumnos y alumnas, que provienen de otros centros, la importancia de valorar a otro alumnado con discapacidades pero que participan como expertos. Esta experiencia, esta práctica educativa, ha favorecido juntar, al tiempo que ha permitido disfrutar en torno a un taller a alumnado diverso, distinto en edad, en procedencia, en religión, en capacidad intelectual, pero en igualdad de posibilidad de avance, de aprendizaje y de fomento de la autoestima. Esto es lo que se pretende con estas actividades desde la educación no formal, que ha sido una gran satisfacción para todos.

Respecto al tercer objetivo que trataba de corroborar si la enseñanza-aprendizaje del patrimonio cultural más próximo, favorece el aprendizaje crítico y significativo para la construcción de una ciudadanía más democrática, constatamos que se ha conseguido, y que los resultados obtenidos nos han demostrado, como indican Domínguez-Almansa, CostaCasais y López-Facal (2019), que el patrimonio cultural puede utilizarse como oportunidad didáctica que lleve al descubrimiento y haga reflexionar y fomentar el espíritu crítico del alumnado, contribuyendo a desarrollar las competencias social y cívica en este, dada cuenta que las culturas están en constante evolución. Llama la atención cómo casi la mitad de los participantes de ascendencia no española encuentran parecidos con las costumbres de sus países de origen. Hay que tener en cuenta que un gran porcentaje son sudamericanos y marroquíes. Estimamos que la educación patrimonial desarrollada en los museos se presenta como una gran aliada para reforzar los conocimientos adquiridos en el aula y formar ciudadanos reflexivos y críticos tal como Fontal-Merillas e Ibáñez-Etxeberría (2015). En la línea de Sales, Moliner, Amiama y Lozano (2018), consideramos importante aunar esfuerzos para llevar a cabo estrategias en las que se prime la colaboración con el entorno y se desarrollen redes sociales para conectar el mayor número posible de agentes educativos, como apuntan Escudero y Martínez (2011). De otro lado, siguiendo a Arnaiz (2012); Brik, Sebring, Allensworth, Luppescu y Easton (2010), creemos positivo favorecer la participación y colaboración de dichas instituciones sociales con los centros escolares locales, al objeto de que estos sean más democráticos e inclusivos. Observamos que los encuestados consideran importante establecer nexos entre entorno y escuela, así como, la cooperación entre esta y otros agentes socioeducativos del municipio (Lozano, Ballesta, Castillo y Cerezo, 2018) con la finalidad de mejorar los procesos de enseñanza-aprendizaje de los alumnos y contribuir al desarrollo socioeducativo e intercultural de los mismos.

\section{Referencias}

Ainscow, M. (2015). Towards self-improving school Systems. Lessons from a city chalenge. Londres: Routledge.

Ainscow, M., Dyson, A., Goldrick, S. y West, M. (2012). Making schools effective for all: rethinking the task. School Leadership \& Management, 32(3), 197-213. DOI:10.1080/13632434.2012.669648

Anderson, K. J. y Minke, K. M. (2007). Parent involvement in education: Toward an understanding of parents' decision making. The Journal of educational research, 100(5),311323.DOI: $10.3200 / J O E R .100 .5 \cdot 311-323$ 
Alves, L.A. y Pinto, H. (2019). Educación histórica con el patrimonio: desafiando la formación del profesorado. REIFOP Revista Electrónica Interuniversitaria de Formación del Profesorado, 22(1), 71-81. DOI:10.6018/reifop.22.1.356381

Arnáiz, P. (2012). Escuelas eficaces e inclusivas: cómo favorecer su desarrollo. Educatio Siglo XXI, 30(1), 25 - 44. https://revistas.um.es/educatio/article/view/149121

Arnaiz, P., De Haro, R. y Azorín, C.M. (2018). Redes de apoyo y colaboracion para la mejora de la educación inclusive. Profesorado. Revista de Currículum y Formación de Profesorado, 22 (2), 2949. doi:10.30827/profesorado.v22i2.7713

Brik, A., Sebring, P. B., Allensworth, E., Luppescu, S. y Easton, J. (2010). Organizing Schools forImprovement. Lessons from Chicago. Chicago: University of Chicago Press.

Cabalé Miranda, E., \& Rodríguez Pérez de Agreda, G. M. (2017). Educación no formal: potencialidades y valor social.Revista Cubana de Educación Superior, 36(1), 69-83. http://scielo.sld.cu/scielo.php?pid=S0257-43142017000100007\&script=sci_arttext\&tlng=pt

Campo-Arias, A., y Oviedo, H. C. (2008). Propiedades psicométricas de una escala: la consistencia $\begin{array}{llll}\text { interna. Rev Salud } \quad \text { Pública, } & 10(5), & \text { pp. } & 831-839 .\end{array}$ https://www.scielosp.org/article/rsap/2008.v10n5/831-839/

Casanova, M.A. (2011). Educación inclusiva: un modelo de futuro. Madrid: WoltersKluwer.

Cerezo, M.C. (2015). La incorporación del alumnado de procedencia extranjera a los centros de Educación Infantil y Primaria de la Región de Murcia [tesis doctoral, Universidad de Murcia]. Repositorio https://digitum.um.es/digitum/handle/10201/47318

Colás, M. P., Buendía, L. y Hernández Pina, F. (2009). Competencias científicas para la realización de una tesis doctoral: Guía metodológica de elaboración y presentación. Barcelona: Editorial Davinci.

Creswell, J. W. (2005).Educational research: Planning, conducting and evaluating quantitative and qualitative research (2. ${ }^{\text {a }}$ ed.). UpperSaddleRiver: Pearson Education.

Domínguez-Almansa, A., Costa-Casais, M. y López-Facal, R. (2019). Educar para reconocer: apropiación patrimonial de los depósitos cuaternarios del litoral gallego por estudiantes de Magisterio. REIFOP Revista Electrónica Interuniversitaria de Formación del Profesorado, 22(1), 5770. DOI: 10.6018/reifop.22.1.357591

Echeíta, G., Simón, C., Sandoval, M. y Monarca, H. (2013).Cómo fomentar las redes naturales de apoyo en el marco de una escuela inclusiva: propuestas prácticas, Sevilla: MAD.

Escudero, J. M. y Martínez, B. (2011). Educación inclusiva y cambio escolar. Revista lberoamericana de Educación, 55, 85 - 105. https://bit.ly/3vn8pld

Etxebarría, F., Karrera, I., y Murua, H. (2010). Competencias interculturales del profesorado con alumnado inmigrante en el País Vasco. REIFOP Revista Electrónica Interuniversitaria de Formación del Profesorado, 13(4), 79-94. https://www.redalyc.org/pdf/2170/217015570007.pdf

Fontal-Merillas, O., y Ibáñez- Etxeberria, A. (2015). Estrategias e instrumentos para la educación patrimonial en España. Educatio Siglo XXI, 33(1), 15-32.DOI: 10.6018/j/222481.

Fontal-Merillas, O. y Ibáñez-Etxeberria, A. (2017). La investigación en educación patrimonial. Evolución y estado actual a través del análisis de indicadores de alto impacto. Revista de Educación (375), 184-214. DOI: 10.4438/1988-592X-RE-2016-375-340 
García-Pérez, E. (2018). Historias de vida: una nueva perspectiva desde la antropología visual [tesis doctoral, Universidad Complutense de Madrid]. Repositorio https://eprints.ucm.es/id/eprint/49493/

Hammersley, M. \& Atkinson, P. (2007). Ethnography: Principles in practice (3. ${ }^{\mathrm{a}}$ ed.). Londres: Routledge.

Jiménez, D., Martínez de Miguel López, S., \& Vizcaíno, J. (2020). El papel de la escuela en la promoción del patrimonio cultural: Un análisis a través del folklore. Revista Electrónica Interuniversitaria De Formación Del Profesorado, 23(3). https://doi.org/10.6018/reifop.384021

Leiva, J. J. y Borrero, R. (Coords.) (2011). Interculturalidad y escuela: perspectiva pedagógica en construcción comunitaria de la escuela intercultural. Barcelona: Octaedro.

Lozano, J., Ballesta, F.J., Castillo, I.S., Cerezo, M.C. (2018). El vínculo de la Escuela con el territorio: una experiencia de inclusión educativa. Profesorado. Revista de currículum y formación del profesorado, 22 (2), 164-182. https://recyt.fecyt.es/index.php/profesorado/article/view/66368

Lozano, J. Ballesta, J., Cerezo, M.C., Luque, F.J., Castillo, I.S. (2017). La participación ciudadana en el centro educativo: las tertulias literarias dialógicas como herramienta para la participación y resolución de conflictos. En A. Palomares (Coord.), Una mirada internacional sobre la educación inclusiva: propuestas de intervención y renovación pedagógica, (pp.371-380). Albacete: Universidad de Albacete.

Lozano, J. y Cerezo, M.C. (2020). El Taller de la Experiencia: creando vínculo intergeneracional. En S. Sales y O. Moliner (Eds.) La escuela incluida en el territorio. La transformación educativa desde la participación ciudadana, (pp.57-84). Barcelona: Octaedro.

Llonch, N. (2011). Enseñar historia: indumentaria y mapas conceptuales. Íber: Didáctica de las ciencias sociales, geografía e historia, 69, 95-101. https://repositori.udl.cat/handle/10459.1/47064

Lucas-Palacios, L. (2014). El patrimonio como herramienta de accesibilidad social. El proyecto patrimonio para involucrarte. En O. Fontal-Merillas, A. Ibáñez-Etxeberria y L. Martín-Sánchez, (Coords.) Reflexionar desde las experiencias.Una visión complementaria entre España, Francia y Brasil.Actas del II Congreso Internacional de Educación y Patrimonio (pp.913-924). Madrid: IPCE/OEPE.

Mager, U. \& Nowak, P. (2012). Effects of student participation in decision making at school. A systematic review and synthesis of empirical research. Educational Research Review, 7 (1), 3861.DOI:10.1016/j.edurev.2011.11.001

Osorio Franco, L. E. (2015). La construcción de la pertenencia socioterritorial: el caso de Jurica, un pueblo al que le llegó la ciudad. Espiral, 22(62), 141-170. http://www.scielo.org.mx/scielo.php?script=sci_arttext\&pid=S1665-05652015000100005

Pérez, G. y Sarrate, M.L. (2013). Diversidad cultural y ciudadanía. Hacia una educación superior inclusiva. Educación XXI, 16 (1), 85-104. DOI: 10.5944/educxx1.16.1.718.

Sales, A., Moliner, O., Amiama, J.F., Lozano, J. (2018). Escuela Incluida: Recursos y estrategias para la participación ciudadana. Revista Mexicana de Investigación, RMIE, 23 (77). http://repositori.uji.es/xmlui/handle/10234/177281

Traver Martí, J. A., Sales Ciges, M. A., Moliner García, M. O., Sanahuja Ribés, A., \& Gil, A. B. (2018). Hacia una Escuela Incluida en su Territorio: Análisis de una práctica comunitaria. Edetania. $\begin{array}{lllll}\text { Estudios } & Y & \text { Propuestas } & \text { Socioeducativas, }\end{array}$ https://revistas.ucv.es/index.php/Edetania/article/view/339 


\section{Anexo I}

\section{Figuras 2 y 3}

Cuestionario para alumnado de Educación Primaria.

DATOS DE IDENTIFICIÓN

Datos correspondientes a su perfil como alumno

1. Marca con una $\mathrm{X}$ en la casilla que correspond

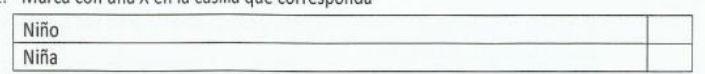

2. ¿Qué edad tienes?

\begin{tabular}{|l|c|}
\hline 6 años & \\
\hline 7 años & \\
\hline 8 años & \\
\hline 9 años & \\
\hline 10 años & \\
\hline 11 años & \\
\hline 12 o más años & \\
\hline Marca con una Xen la casilla correcta & \\
\hline Tu familia: \\
\hline 2.Ha vivido siempre en España & \\
\hline Marca con una Xen $X$ la casilla correcta & \\
\hline
\end{tabular}

${ }^{*}$ Si tu familia ha llegado de otro país, ¿podrías escribir el país de procedencia?

\section{DIMENSIÓN I}

EDUCACIÓN NO FORMAL COMPLEMENTARIA DE LA FORMAL

1. ¿Qué te parece esta actividad fuera del colegio?

\begin{tabular}{|l|l|l|l|}
\hline Muy buena & Buena & Regular & Mala \\
\hline
\end{tabular}

2. ¿Piensas que con actividades como esta se puede aprender más fácilmente que con los libros y demás materiales que se utilizan en las aulas?

\begin{tabular}{|l|l|l|l|}
\hline Siempre & A veces & Casi nunca & Nunca \\
\hline
\end{tabular}

DIMENSIÓN II

ACTIVIDADES EXTRAESCOLARES PARA DESARROLLAR PRÁCTICAS INCLUSIVAS Y CONSTRUIR COMUNIDAD.

3. ¿Te gusta trabajar en equipo?

\begin{tabular}{|l|l|l|l|}
\hline Siempre & A veces & Casi nunca & Nunca \\
\hline
\end{tabular}

4. ¿Crees que trabajar en equipo ayuda a aprender mientras nos divertimos?

\begin{tabular}{|l|l|l|l|}
\hline Siempre & A veces & Casi nunca & Nunca \\
\hline
\end{tabular}

5. Si te toca ir en un equipo con compañeros y compañeras que no son tus mejores amigos o amigas, ¿̇te importa?

\begin{tabular}{|l|l|l|l|}
\hline Mucho & Regular & Poco & Muy poco \\
\hline
\end{tabular}

6. ¿Crees que las actividadesy salidas que se realizan fuera del centroescolar favorecen conocer mejor a los compañeros del colegio?

\begin{tabular}{|l|l|l|l|}
\hline Siempre & A veces & Casi nunca & Nunca \\
\hline
\end{tabular}

DIMENSIÓN III

ACTIVIDADES EXTRAESCOLARES Y EDUCACIÓN PATRIMONIAL PARA CONSTRUIR UNA CIUDADANÍA INTERCULTURAL-INCLUSIVA

7. ¿Conocías las costumbres de tus antepasados (bisabuelos, tatarabuelos...)? \begin{tabular}{|l|l|l|l|}
\hline Mucho & Regular & Poco & Muypoco \\
\hline
\end{tabular}

8. ¿Te ha gustado conocer las tradiciones y costumbres de tus antepasados? \begin{tabular}{|l|l|l|l|}
\hline Mucho & Regular & Poco & Muy poco \\
\hline
\end{tabular}

9. ¿Sabias que en Murcia habian vivido otras culturas como iberos, romanosy árabes?

\begin{tabular}{|l|l|l|l|}
\hline árabes? & Regular & Poco & Muy poco \\
\hline
\end{tabular}

10. ¿Te gusta saber sobre otras culturas diferentes a la tuya?

\begin{tabular}{|l|l|l|l|}
\hline Mucho & Regular & Poco & Muy poco \\
\hline
\end{tabular}

*CONTESTA las siguientes preguntas, Sólo si tus padres y familiares NO SON DE ORIGEN ESPAÑOL:

11. ¿Has encontrado algún parecido con las costumbres de tu país de origen?

\begin{tabular}{|l|l|l|l|}
\hline Muchos & Algunos & Pocos & Ninguno \\
\hline
\end{tabular}

12. ¿Podrias escribir aquellas costumbres idénticas y diferentes que máste han llamado la atención?

Costumbresidénticas

Costumbres diferentes 


\section{Anexo II}

Figuras 4, 5, 6 y 7

Cuestionario para alumnado de Educación Infantil o con dificultades en lectoescritura

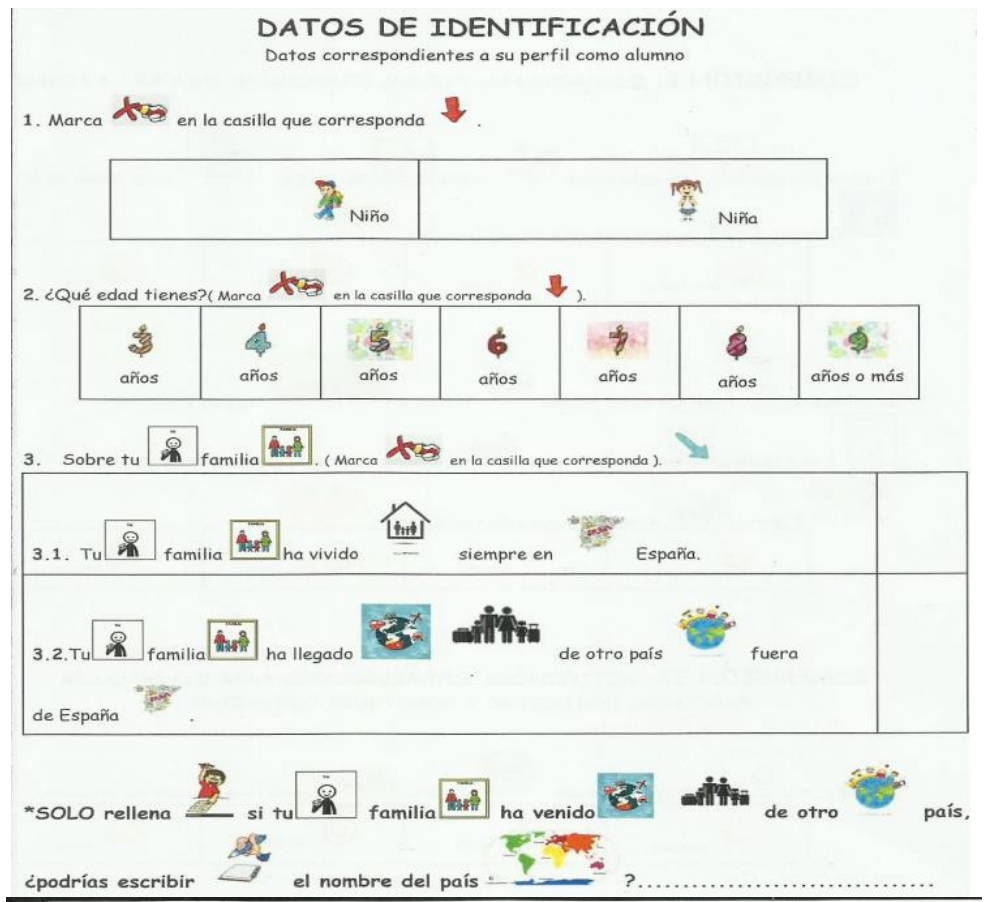

DIMENSIÓN I. EDUCACIÓN NO FORMAL COMPLEMENTARIA DE LA FORMAL

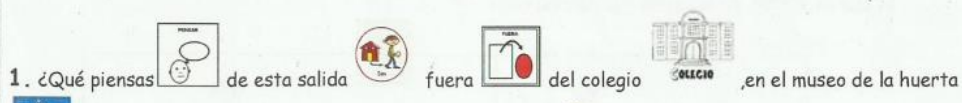

A.
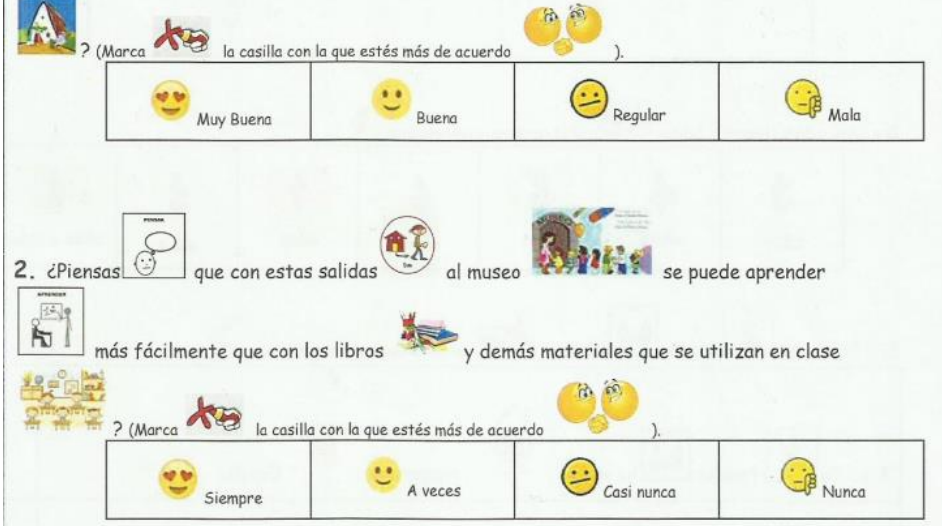

DIMENSIÓN II. ACTIVIDADES EXTRAESCOLARES PARA DESARROLLAR PRÁCTICAS INCLUSIVAS Y CONSTRUIR COMUNIDAD.

3. LTe gusta
\begin{tabular}{|c|c|c|c|}
\hline$\vartheta_{\text {Siempre }}$ & $\ddot{*}_{\text {A veces }}$ & $\overbrace{\text { Casi nunca }}$ & $\overbrace{\text { trabajar en equipo }}$ \\
\hline
\end{tabular} 
Bienvenida García Martínez, Josefina Lozano Martínez \& Mari Carmen Cerezo Maiquez.

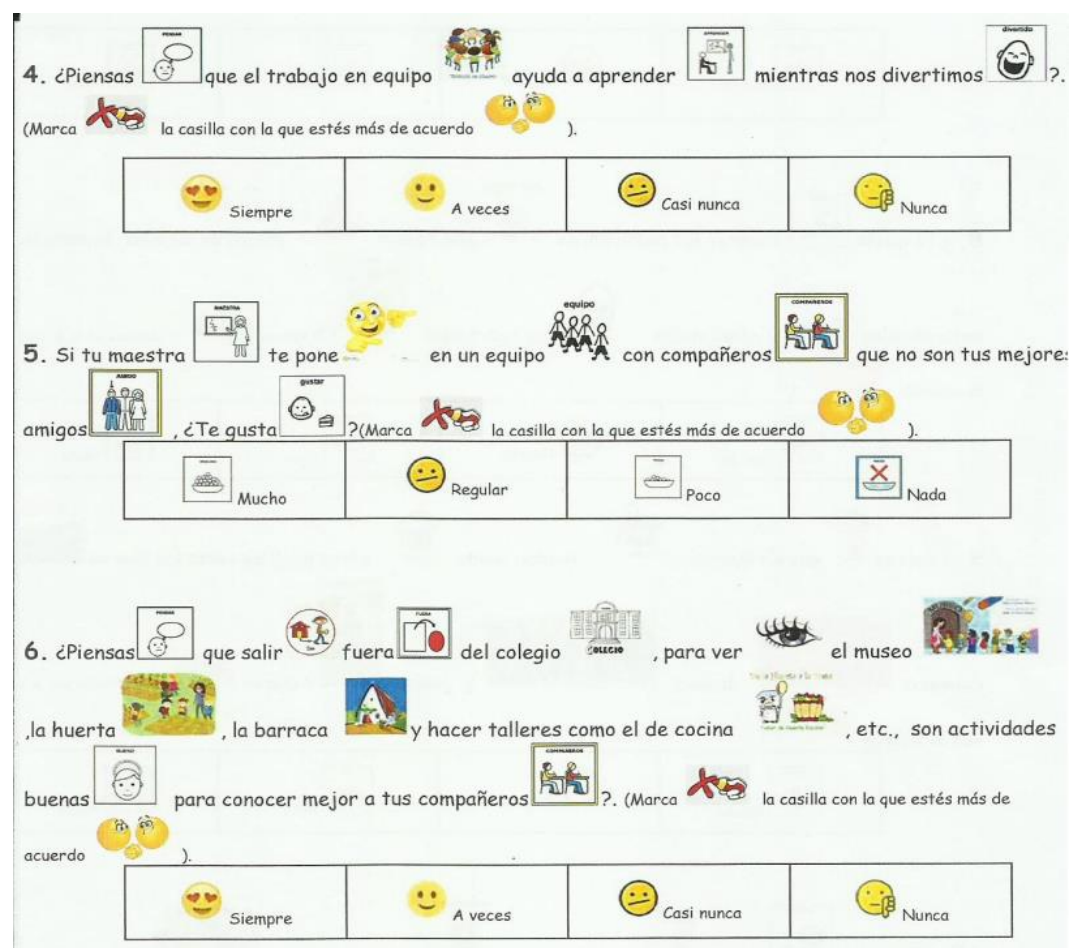

DIMENSIÓN III. ACTIVIDADES EXTRAESCOLARES Y EDUCACIÓN PATRIMONIAL PARA CONSTRUIR UNA CIUDADANÍA INTERCULTURAL-INCLUSIVA

7. ¿Habías oído hablar in sobre abuelos, bisabuelos, tatarabuelos, etc. it it (Marca la casilla con la que estés más de acuerdo (i) ).
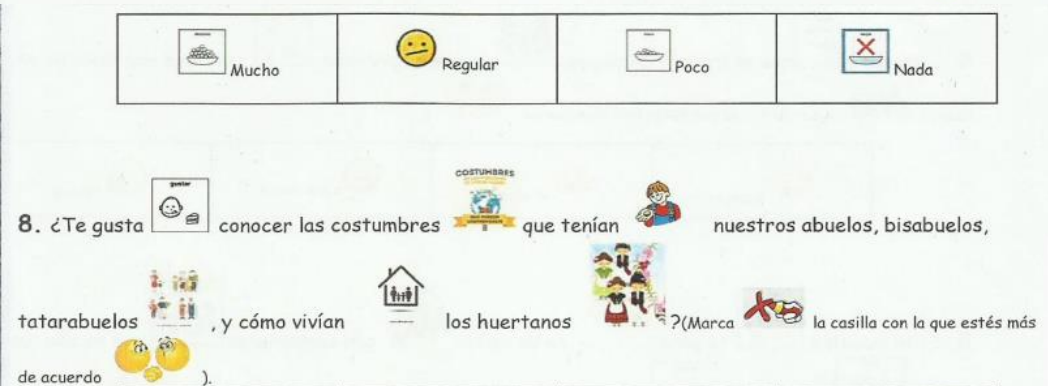
de acuerdo

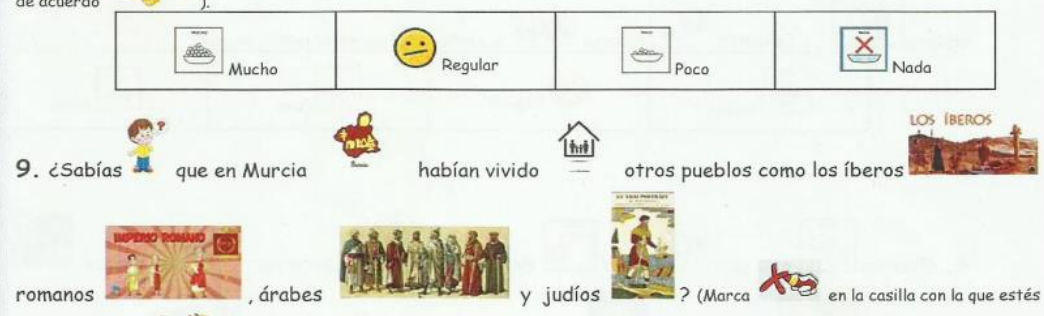
más de acuerdo
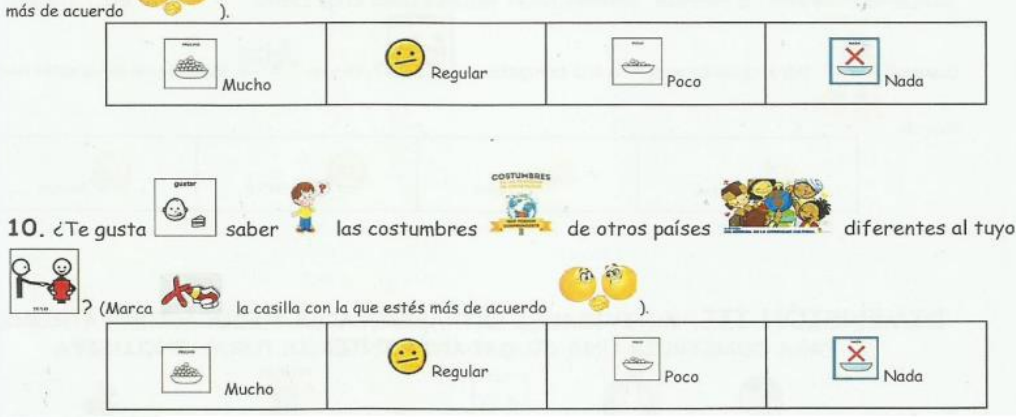

200

Revista Electrónica Interuniversitaria de Formación del Profesorado (REIFOP) 


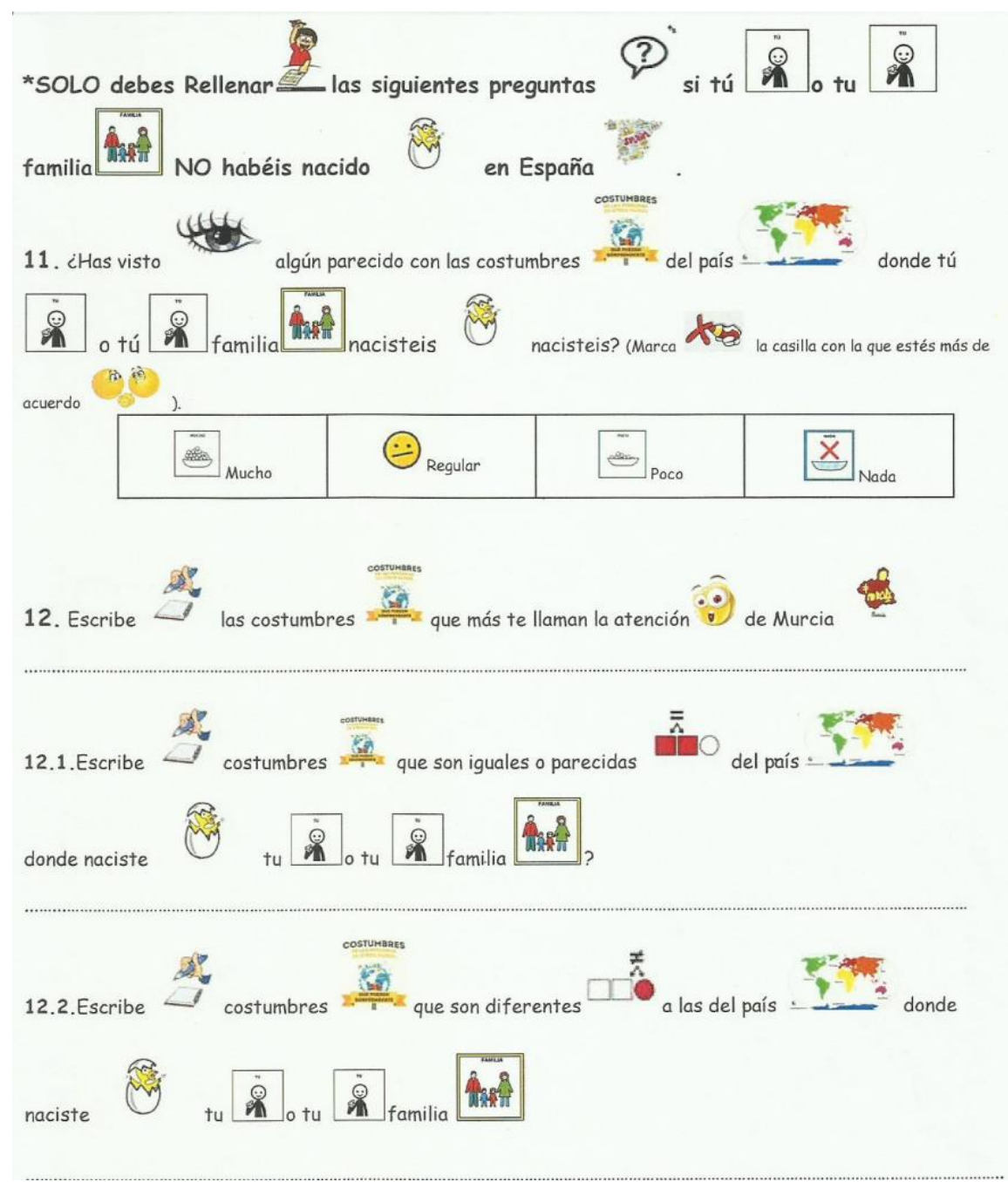

